\title{
Spinal cord protection in surgical and endovascular repair of thoracoabdominal aortic disease
}

\author{
Randall B. Griepp, MD, and Eva B. Griepp, MD
}

In the last decades of the 20th century, as improved cardiovascular techniques allowed more extensive aortic procedures and an aging population increased the number of individuals with symptomatic and life-threatening aortic disease, cardiac and vascular surgeons-and subsequently interventionalists - began to address the repair of extensive aortic disease. As distal aorta procedures became more extensive, spinal cord injury (SCI) began to emerge as a major impediment to success.

In 1993, a report of the extensive experience of Crawford and colleagues ${ }^{1}$ documented an incidence of SCI as high as $30 \%$ when an entire thoracoabdominal aorta was resected. The conventional wisdom was that this was a consequence of disconnecting a segmental vessel input that was the major source of midcord supply: the arteria radicularis magna (also known as artery of Adamkiewicz). Notwithstanding the logical flaw in this premise-in $70 \%$ of cases of total thoracoabdominal resection the spinal cord survives-almost all efforts to reduce the incidence of SCI were directed toward identifying and preserving this important artery, or blindly preserving segmental arteries statistically most likely to give rise to such a vessel. Further clinical experience made it apparent that identifying this vessel physiologically was often difficult, that SCI correlated closely with the number of segmental arteries (ie, intercostal and lumbar arteries) sacrificed rather than their location, and that other factors were important determinants of SCI. In the past few years, laboratory and clinical experience have led to an appreciation of the multifactorial components contributing to SCI, and to a significant reduction in its incidence.

\section{TYPES OF ISCHEMIC SPINAL CORD INJURY}

In the context of aortic procedures, there are 2 major types of SCI. The first is the temporary interruption of cord blood supply for an interval sufficient to cause

\footnotetext{
From the Department of Cardiothoracic Surgery, Mount Sinai School of Medicine, New York, NY.

Disclosures: Authors have nothing to disclose with regard to commercial support. Read at The American Association for Thoracic Surgery Aortic Symposium, New York, New York, April 24-25, 2014.

Received for publication May 2, 2014; revisions received Sept 29, 2014; accepted for publication Oct 6, 2014; available ahead of print Nov 7, 2014

Address for reprints: Eva B. Griepp, MD, Department of Cardiothoracic Surgery, Mount Sinai School of Medicine, 1 Gustav Levy Place, New York, NY 10029 (E-mail: ebgriepp@aol.com).

J Thorac Cardiovasc Surg 2015;149:S86-90

$0022-5223 / \$ 36.00$

Copyright (C) 2015 by The American Association for Thoracic Surgery

http://dx.doi.org/10.1016/j.jtcvs.2014.10.056
}

irreversible ischemic necrosis. The most frequent cause is the clamping of the proximal thoracoabdominal aorta without any distal perfusion. In experimental animals, clamping the aorta just distal to the left subclavian artery results in a complete failure of cord perfusion below T10, and clinical experience suggest this is also true in humans. ${ }^{2}$ This distal ischemia can be attenuated by hypothermia and by perfusion of the hypogastric arteries and distal lumbar vessels.

The second type of SCI results from the permanent interruption of sufficient sources of cord blood supply to render a portion of the cord nonviable. These sources include the segmental arteries and the subclavian and hypogastric arteries.

Finally, a combination of these types of cord injury can occur: sublethal ischemic injury caused by aortic clamping followed by borderline postoperative blood supply secondary to segmental vessel sacrifice. A period of unstable hemodynamics may also play a role in augmenting the risk of SCI.

\section{GENERALLY ACCEPTED PRINCIPLES FOR MINIMIZING SPINAL CORD ISCHEMIA}

A number of generally accepted principles for spinal cord protection for both surgical and endovascular treatment of thoracoabdominal aortic disease have been established based on clinical experience, controlled clinical studies, and laboratory investigations in animal models. The first is the powerful influence of hypothermia in increasing the interval of safe cord ischemia. With aortic clamping distal to the left subclavian artery, spinal cord perfusion is absent below T10. Full clinical recovery is possible after a maximum of 20 minutes at $37^{\circ} \mathrm{C}$, but the safe ischemic interval can be increased substantially at lower temperatures. The Q10 of cord neurons is approximately 2.3. Because cord metabolism is slowed by hypothermia, this means that the safe ischemic interval is prolonged by a factor of 2.3 for every $10^{\circ} \mathrm{C}$ drop in temperature. ${ }^{3}$ Almost all surgeons currently perform aortic resections at a temperature of $30^{\circ} \mathrm{C}$ to $32^{\circ} \mathrm{C}$, and a number prefer to perform extensive aortic resections at $15^{\circ} \mathrm{C}$ to $20^{\circ} \mathrm{C}$, utilizing cardiopulmonary bypass and circulatory arrest. $^{4}$

An appreciation of the interconnectedness of the components of circulatory input into cord blood supply has gradually become apparent, including awareness of the importance of nonsegmental vessels, primarily the 

Abbreviations and Acronyms
$\mathrm{CSF}=$ cerebrospinal fluid
$\mathrm{SCI}=$ spinal cord injury
$\mathrm{SCPP}=$ spinal cord perfusion pressure

subclavian and hypogastric arteries. In experimental animals, segmental artery sacrifice is much better tolerated if the subclavian and hypogastric arteries are patent. ${ }^{5}$ Two recent clinical studies document that occlusion of the subclavian and/or hypogastric arteries increases the risk of SCI in surgical and endovascular procedures on thoracoabdominal aortas. ${ }^{6,7}$

Use of distal clamping of the aorta during surgical resections, with perfusion of the pelvic/lower abdominal circulatory beds by left atrial-femoral artery bypass or partial cardiopulmonary bypass, has been documented to reduce the incidence of SCI in a number of clinical series. ${ }^{8}$ Direct measurements of cord perfusion pressure have documented the efficacy of distal perfusion, but have also revealed that nonpulsatile perfusion is not as effective as pulsatile perfusion at the same mean pressure. $^{9}$

Drainage of cerebrospinal fluid (CSF) during and after both surgical and endovascular procedures is now used routinely when the risk of SCI is thought to be significant. Acceptance of the efficacy of CSF drainage has rested chiefly on ubiquitous anecdotal evidence that it can reverse delayed paraplegia/paraparesis, but there is also a randomized prospective study in patients with surgical resection of thoracoabdominal aneurysms which confirms its benefit. ${ }^{10}$

In a similar paradigm, episodes of delayed paraplegia/ paraparesis have frequently been associated with periods of hemodynamic instability, and have been reversed by increasing blood pressure and cardiac output. Clearly, both an increase in arterial pressure and a decrease in CSF pressure result in a net increase in perfusion of the spinal cord. Similarly, because central venous pressure and CSF pressure are-at least in part-additive in increasing outflow pressure from the spinal canal, it is important to avoid hemodynamic management strategies that entail high venous pressure.

Because the adequacy of spinal cord perfusion-and consequently cord viability - may vary considerably during and immediately after surgical and endovascular procedures, monitoring of cord health is important for both interventional and anesthetic management. Lower limb function cannot be evaluated in the anesthetized or sedated patient, so one must rely upon indirect assessment. Presently available alternatives are somatosensory evoked potentials and motor evoked potentials. Both are somewhat time-consuming and burdensome, but they have been shown to be useful in patient management, and they are probably important in the development of team expertise. ${ }^{11}$

\section{DEVELOPMENT OF THE COLLATERAL NETWORK CONCEPT}

For the past decade or more, we have studied the anatomy and physiology of spinal cord circulation and its response to sacrifice of segmental arteries to formulate additional strategies to reduce the incidence of SCI following repair of thoracoabdominal aneurysms. These studies were carried out in a juvenile pig model because the spinal cord circulation in pigs is very similar to that in human beings.

Anatomic studies were done by injecting the circulation of the pigs with an acrylic resin, which after digestion of the surrounding tissue reveals a cast of the circulation. Additional anatomic studies in pigs that had undergone vessel sacrifice were studied by injection studies with a barium-latex emulsion ${ }^{12,13}$; visualization was accomplished with postmortem high-resolution computed tomography scans. The initial anatomic studies led to the revelation that the anterior spinal artery is richly interconnected with the vascular supply of the adjacent musculature of the back, whose volume dwarfs that of the vessels going to the cord. There are multiple vascular connections at each segmental level between adjacent muscle and the blood supply of the cord, and also multiple ways in which the intraspinal vasculature at each segmental level is connected with the levels above and below it (Figure 1). The multilevel intraspinal vascular network is also clearly connected to the subclavian and hypogastric arteries, and via the intercostal vessels, to the internal thoracic arteries. This extensive vascular network has the potential to compensate for loss of input to the spinal cord from a single and even multiple sources by drawing upon alternative vessels that remain patent, providing flow even from some distance away. When overall flow into the network is reduced, experimental evidence shows that blood is preferentially directed to the spinal cord rather than to adjacent ischemic muscle.

How the collateral circulation adapts functionally has been revealed by a number of physiologic studies in juvenile pigs. ${ }^{14,15}$ In this model, not only can the vascular consequences of various thoracoabdominal repairs be simulated, but also pressures (and flows) can be measured, spinal cord function can be assessed directly and indirectly, histologic changes can be quantitated, and postoperative behavioral recovery can be monitored for a week or longer. By inserting a pressure catheter in the distal stump of a divided lumbar artery, one can monitor the recovery of spinal cord circulation following sacrifice of variable numbers of segmental arteries in simulated repairs of various extents and types. Using this methodology, we discovered that spinal cord perfusion 


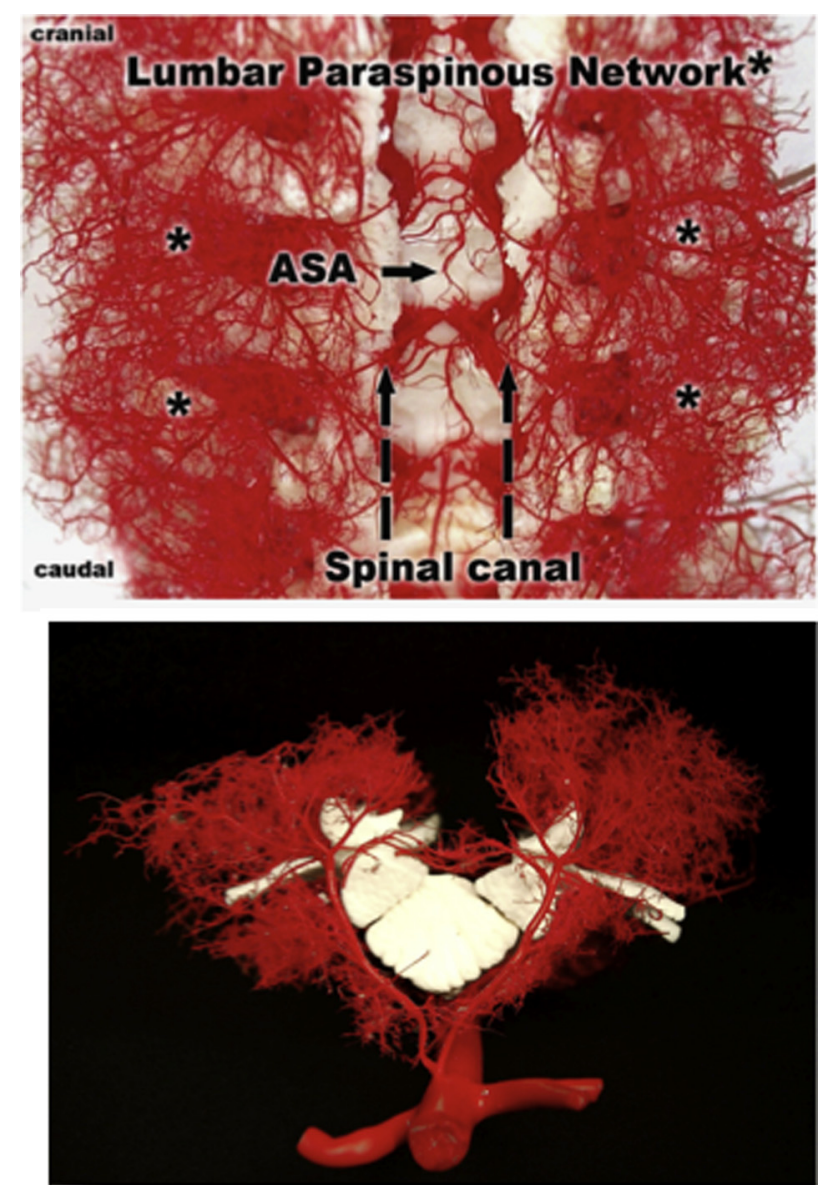

FIGURE 1. Acrylic casts of the collateral network available for spinal cord perfusion in a pig. Top, Dorsal view of multiple lumbar levels demonstrating the anterior spinal artery (ASA; arrow) and its multiple connections at each level with intra- and extrathecal vessels. *Lumbar paraspinous network. Bottom, Transverse section at the level of L1, demonstrating the disparity between the neural and the muscular vascularization. Reprinted with permission. ${ }^{12}$

pressure (SCPP) - the measured pressure in the collateral network-is about $80 \%$ of mean arterial pressure at baseline, and drops gradually during serial sacrifice of segmental arteries to about $25 \%$ of baseline when all segmental arteries are sacrificed. Approximately half the pigs develop SCI postoperatively if all the segmental arteries are sacrificed. It is notable that all the pigs-even those who sustain SCI-show return of SCPP to baseline levels by 5 days after simulated thoracoabdominal aneurysm repair.

The stability of SCPP by 5 days after even very extensive segmental arteries sacrifice seems-based on the evidence of anatomic studies-to come from a combination of an increase in the size of existing small arterioles within the collateral network, and the development of new small vessels within it. ${ }^{16}$ The cumulative effect of extensive segmental artery sacrifice on the anatomy of the collateral network can be visualized in radiographs of pigs injected with the barium latex emulsion 5 days after extensive segmental artery sacrifice (Figure 2).

The reliable recovery of SCPP a week after even extensive segmental artery sacrifice suggested that it might be possible to mitigate the detrimental effect of the extensive loss of segmental artery input if the procedure was done in stages, allowing the collateral circulation to adapt and stabilize following partial loss of segmental artery input during the first stage. We undertook a series of experiments that documented that the drop in SCPP is in fact much less pronounced during the second stage of a 2 -stage procedure: rather than falling to $25 \%$ of baseline, the pressures fall to only $40 \%$ of baseline during the second stage, regardless of whether the initial sacrifice of segmental arteries was in the thoracic aorta or in the lumbar aorta. ${ }^{9,17}$ The results of the 2-stage approach do not differ significantly when occlusion of the segmental artery is achieved using a stent compared with open surgical sacrifice. ${ }^{18}$

The less severe drop in SCPP following extensive segmental artery sacrifice carried out in 2 stages is accompanied by a highly significant difference in outcome: none of the animals in the 2-stage group experienced SCI. This result echoed observations from a retrospective clinical study of 90 patients in whom all or nearly all segmental arteries had been ligated: none of the 35 patients with extensive loss of segmental arteries as a result of 2 procedures developed functional SCI, in contrast with 8 of 55 patients who had a slightly less extensive resection in a single operation $(P<.02) .{ }^{19}$ A lower rate of SCI following 2-stage versus single-stage aneurysm repair is also evident in a series of patients undergoing repair of Crawford type II thoracoabdominal aneurysms reported by Coselli and colleagues. ${ }^{20}$ Troisi and colleagues ${ }^{21}$ pursued a deliberately staged hybrid strategy to replace the entire thoracoabdominal aorta in 12 patients, none of whom developed SCI. No cases of permanent paraplegia were observed when Harrison and colleagues ${ }^{22}$ treated 10 patients with Crawford type II thoracoabdominal aneurysms with branched graft repair in whom sac perfusion was maintained for 7 to 10 days with an open side graft that was then closed.

The 2-stage approach to repair of extensive thoracoabdominal aneurysms was not carried out by design in many of the patients included in the clinical reports, and one can imagine that a 2-stage protocol may not be feasible in all patients. We thus undertook to see whether one could provoke the changes in the collateral network that occur after the first stage of a 2-stage protocol by electively occluding a few segmental arteries 1 week before extensive segmental artery sacrifice using endovascular coils. ${ }^{23}$ In this experimental protocol, 3 groups of 10 pigs each underwent surgical ligation of the lumbar arteries followed by 


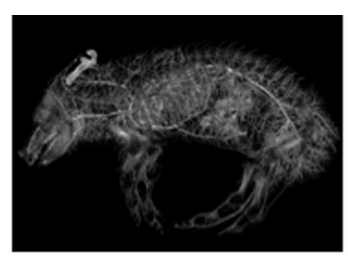

Normal

Five days after total segmental vessel ligation
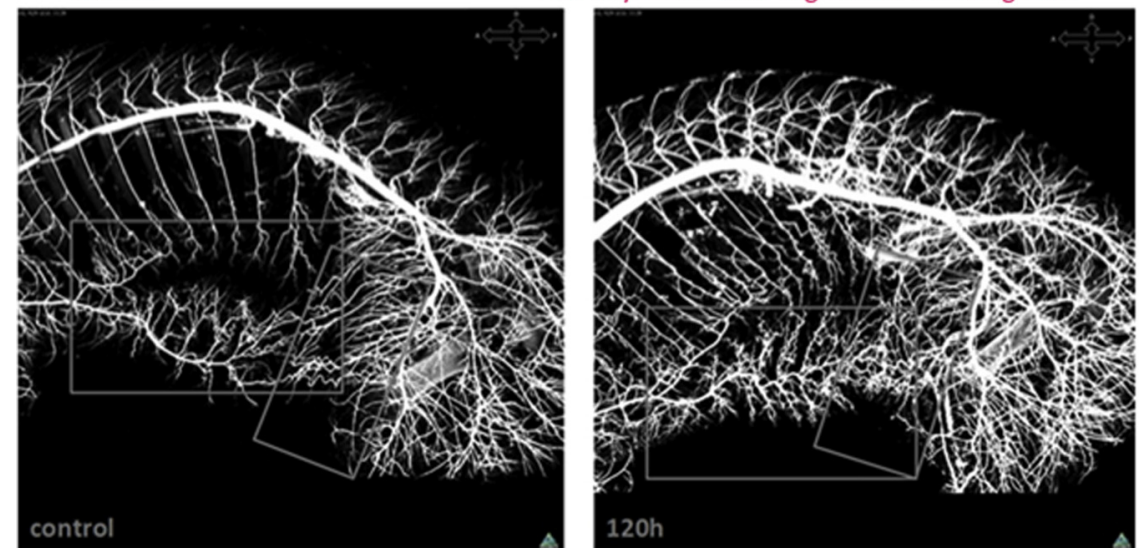

FIGURE 2. Porcine vasculature demonstrated by radiograph following latex barium emulsion infusion. Top, Entire pig. Bottom left, Eviscerated trunk of a normal pig. Bottom right, Eviscerated trunk of a pig 5 days after ligation of all segmental vessels. Note neovascularization connecting distal segmental vessels with the sacral vessels and the subclavian vessels via the internal thoracic arteries, as well as the complete backfilling of the segmental vessels to the ligation sites adjacent to the aorta. Reprinted with permission. ${ }^{13}$

occlusion of the intercostal arteries by thoracic stenting (complete segmental artery sacrifice). In the control group, segmental artery sacrifice was carried out without any prior procedure; in the second group, 1 to 2 lumbar or lower thoracic arteries were occluded by a vascular coil 1 week before segmental artery sacrifice, and in the third group, 4 to 5 segmental arteries were coiled during the preliminary procedure. Whereas 6 out of 10 pigs developed paraplegia or paraparesis in the control group (as expected), no pigs in the group with 4 or 5 segmental arteries coiled during the preliminary procedure had functional SCI postoperatively, and only 2 out of 10 pigs developed paraplegia after 1 or 2 arteries were occluded during the preliminary procedure. Postmortem examination of the spinal cords confirmed an absence of severe histopathologic injury in pigs with several segmental arteries coiled before extensive segmental artery sacrifice, in contrast with significant histopathology in the controls (ie, without preliminary coiling).

\section{CONCLUSIONS}

The improved understanding of the collateral network revealed by recent clinical and experimental studies has provided a rationale for some of the proposed recommendations for optimal management of repair of extensive thoracoabdominal aneurysms aimed to minimize paraplegia. The 2-stage concept may allow safe repair of even extensive aneurysms in elective cases. The option of preliminary occlusion of some segmental arteries before surgical or interventional repair of extensive aneurysms may enhance the safety of repair in some additional patients not suitable for a more conventional 2-stage approach. Together with improvements in intraoperative spinal cord protection and enhanced vigilance to avoid hemodynamic instability while spinal cord circulation is adapting to loss of input postoperatively, the collateral network strategy should allow us to attain the goal of eliminating almost all cases of SCI following thoracoabdominal aneurysm repair.

\section{References}

1. Svensson LG, Crawford ES, Hess KR, Coselli JS, Safi HJ. Experience with 1509 patients undergoing thoracoabdominal operations. J Vasc Surg. 1993;17:357-68; discussion 368-70

2. Etz CD, Luehr M, Kari FA, Lin HM, Kleinman G, Zoli S, et al. Selective cerebral perfusion at $28^{\circ} \mathrm{C}$-is the spinal cord safe? Eur J Cardiothorac Surg. 2009;36: 946-55.

3. Griepp RB, Di Luozzo G. Hypothermia for aortic surgery. J Thorac Cardiovasc Surg. 2013;145(Suppl):S56-8.

4. Kulik A, Castner CF, Kouchoukos NT. Outcomes after thoracoabdominal aortic aneurysm repair with hypothermic circulatory arrest. J Thorac Cardiovasc Surg. 2011;141:953-60.

5. Strauch JT, Spielvogel D, Lauten A, Zhang N, Shiang H, Weisz D, et al. Importance of extrasegmental vessels for spinal cord blood supply in a chronic porcine model. Rev Port Cir Cardiotorac Vasc. 2003;10:185-91.

6. Czerny M, Eggebrecht H, Sodek G, Verzini F, Cao P, Maritati G, et al. Mechanisms of symptomatic spinal cord ischemia after TEVAR: insights from the European Registry of Endovascular Aortic Repair Complications. J Endovasc Ther. 2012;19:37-43.

7. Eagleton MJ, Shah S, Petkosevek D, Mastracci TM, Greenberg RK Hypogastric and subclavian artery patency affects onset and recovery of 
spinal cord ischemia associated with aortic endografting. J Vasc Surg. 2014;59: 89-95.

8. Estrera AL, Miller CC, Chen EP, Meada R, Torres RH, Porat EE, et al. Descending thoracic aortic aneurysm repair: 12 year experience using distal aortic perfusion and cerebrospinal fluid drainage. Ann Thorac Surg. 2005;80: 1290-6.

9. Etz CD, Zoli S, Bischoff MS, Bodian C, Di Luozzo G, Griepp RB. Measuring the collateral network pressure to minimize paraplegia risk in thoracoabdominal aneurysm resection. J Thorac Cardiovasc Surg. 2010;140(Suppl):S125-30; discussion S142-6.

10. Coselli JS, LeMaire SA, Koksoy C, Schmittling ZC, Curling PE. Cerebrospinal fluid drainage reduces paraplegia after thoracoabdominal aortic aneurysm repair: results of a randomized clinical trial. J Vasc Surg. 2002;35:631-9.

11. Estrera AL, Sheinbaum R, Miller CC, Harrison R, Safi HJ. Neuromonitor-guided repair of thoracoabdominal aortic aneurysms. J Thorac Cardiovasc Surg. 2010; 140(Suppl):S131-5; discussion S142-6.

12. Etz CD, Kari FA, Mueller CS, Silovitz D, Brenner RM, Lin HM, et al. The collateral network concept: a reassessment of the anatomy of spinal cord perfusion. J Thorac Cardiovasc Surg. 2011;141:1020-8.

13. Geisbuesch S, Bischoff MS, Lin HM, Griepp RB, Di Luozzo G. Imaging of vascular remodeling following simulated thoracoabdominal aneurysm repair. J Thorac Cardiovasc Surg. 2012;144:1471-8.

14. Etz CD, Homann TM, Plestis KA, Zhang N, Luehr M, Weisz DJ, et al. Spinal cord perfusion after extensive segmental artery sacrifice: can paraplegia be prevented? Eur J Cariodthorac Surg. 2007;31:643-6.

15. Etz CD, Homann TM, Luehr M, Kari FA, Weisz DJ, Kleinman G, et al. Spinal cord blood flow and ischemic injury after experimental sacrifice of thoracic and abdominal segmental arteries. Eur J Cadiothorac Surg. 2008;33:1030-8.
16. Etz CD, Kari FA, Mueller CS, Silovitz D, Brenner RM, Lin HM, et al. The collateral netowrk concept: remodeling of the arterial collateral circulation after experimental segmental artery sacrifice. J Thorac Cardiovasc Surg. 2011; 141:1029-36.

17. Zoli S, Etz CD, Roder F, Brenner RM, Bodian CA, Kleinman G, et al. Experimental two-stage simulated repair of extensive thoracoabdominal aneurysms reduces paraplegia risk. Ann Thorac Surg. 2010;90:722-9.

18. Bischoff MS, Scheumann J, Brenner RM, Ladage D, Bodian CA, Kleinman G, et al. Staged approach prevents spinal cord injury in hybrid surgicalendovascular thoracoabdominal aortic aneurysm repair: an experimental model. Ann Thorac Surg. 2011;92:138-46.

19. Etz CD, Zoli S, Mueller CS, Bodian CA, Di Luozzo G, Lazala R, et al. Staged repair significantly reduces paraplegia rate after extensive thoracoabdominal aortic aneurysm repair. J Thorac Cardiovasc Surg. 2010;139: 1464-72.

20. Coselli JS, Poli dE, Figueiredo LF, LeMaire SA. Impact of previous thoracic aneurysm repair on thoracoabdominal aortic aneurysm management. Ann Thorac Surg. 1997;64:639-50.

21. Troisi N, Bichi S, Patrini D, Arena V, Setti M, Piti A, et al. Hybrid three stage repair of mega aortic syndrome with the Lupiae technique. J Thorac Cardiovasc Surg. 2013;145(Suppl):S171-7.

22. Harrison SC, Agu O, Harris PL, Ivancev K. Elective sac perfusion to reduce the risk of neurologic events following endovascular repair of thoracoabdominal aneurysms. J Vasc Surg. 2012;55:1202-5.

23. Geisbuesch S, Stefanovic A, Korsuth JS, Lin HM, Morgello S, Weisz DJ, et al. Endovascular coil embolization of segmental arteries prevents paraplegia after subsequent thoracoabdominal repair: an experimental model. J Thorac Cardiovasc Surg. 2014;147:220-7. 